\title{
One Week, Many Ripples: Measuring the Impacts of the Fall Reading Week on Student Stress
}

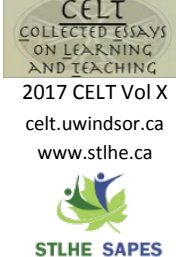

\author{
Heather Poole, Ayesha Khan, and Michael Agnew \\ McMaster University
}

DOI: $10.22329 /$ celt.v10i0.4757

More and more Canadian post-secondary institutions are introducing a fall break into their term calendars. In 2015, a full week fall break was introduced at our university in order to enhance academic performance and improve mental health amongst students. Our interdisciplinary team surveyed undergraduate students at our university about their experience of the fall break, collected standardized measures of experienced stressors and perceptions of stress before and after the break, and hosted several focus groups to develop a detailed narrative of students' experience. Stress can also be assessed through non-invasive hormone measures. We collected saliva samples to profile metabolic hormones, cortisol, and dehydroepiandrosterone (DHEA), from first-year male engineering students in order to document possible changes in their stress levels before and after the week-long break. This group was compared to male engineering students at a similar university that does not hold a fall break. Students exhibited a lower ratio of cortisol to DHEA after a fall break than those that did not experience a break. Our survey results indicate that the majority of students thought the fall break was a positive experience. However, self-reports of stress show a more complex picture, with many students reporting increased perceived stress after the break. Additionally, a portion of students reported that the fall break was a negative experience. To the best of our knowledge, our study is the first of its kind to use a mixed-methods approach to examine the impacts of a fall break.

$\mathrm{T}$ he mental health and wellness of university and college students has received recent attention as popular media reports and empirical research indicates increased rates of stress and mental illness in this group (Booth, Sharma, \& Leader, 2015; Chiose, 2016; Lunau, 2012). Indeed, post-secondary students have long reported higher levels of stress than the general population (Adlaf, Gliksman, Demers, \& Newton-Taylor, 2001). In 2013, a large-scale survey assessed well-being across 30 post-secondary institutions in Canada (American College Health Association, 2013). A total of $89.3 \%$ of respondents felt overwhelmed, $86.9 \%$ felt exhausted, and $56.5 \%$ felt immense anxiety. A majority of students (56.6\%) reported academic issues as traumatic or very difficult to handle. A larger follow-up survey in 2016 captured similar responses from 43,000 students in 41 Canadian institutions (Canadian Association of College and University Student Services, 2016). These survey data support previous trends indicating that the mental health of our students is in crisis. Anxiety and depression were reported to be the most common mental illnesses experienced by students. Moreover, $13 \%$ of survey respondents had considered suicide within the last 12 months: a 3.5\% increase from 2013 (Canadian Association of College and University Student Services, 2016). Although alarming, these data are not surprising. Post- 
secondary students are part of a special cohort, since onset of mental illness commonly occurs between the ages of fifteen and twenty-four (Eisenberg, Gollust, Golberstein, \& Hefner, 2007; Rao, Hammen, \& Daley, 1999), and the stressors affecting their mental health may be different than those impacting their non-student counterparts or others in a different lifestage.

According to current estimates, nearly 2 million students are registered at post-secondary institutions across Canada (Statistics Canada, 2016; Universities Canada, 2016). Colleges and universities are now paying special attention to the mental wellbeing of their students. Multiple initiatives aim to educate students and facilitate discussion about emotional and physical health (e.g., COPE, http://copex.weebly.com; Healthy Minds at UBC, http://blogs.ubc.ca/healthyminds/; More Feet on the Ground, https://brock.morefeetontheground.ca), and to train staff and faculty to meet the needs of students (e.g., Green Folder, http://www.queensu.ca/ studentwellness/mental-health). Governments and student organizations are also developing programs to support mental health (e.g., Bartlett, 2014; Centre for Innovation in Campus Mental Health, http://campusmentalhealth.ca/projects/;

http://www.good2talk.ca).

Following a year in which several students died by suicide, Queen's University developed a task force to discuss student mental health. The introduction of a fall break was a major recommendation from the group (Clapham, Jahchan, Medves, Tierney, \& Walker, 2012). Other universities held related discussions, leading to the consensus that a mid-semester break in both fall and winter would support student mental health and increase well-being (e.g., Cramer \& Pschibul, 2017; McMaster Daily News, 2015). Accordingly, the trend of holding a mid-semester fall break is growing: upon a recent review of the websites of 70 Canadian universities, 49 indicated that some form of a multiday break is scheduled for the 2016 fall term.

Given the wide-scale adoption of fall breaks as a means to reduce student stress, it is critical to investigate whether this intervention improves students' mental health. There is some work investigating this: students at Brock University self- reported decreased stress due to a fall break (Pilato, 2014) and researchers at the University of Windsor using smartphone notifications to track how students spent their time found that post-break stress correlated with pre-break stress, workload, and recreational activities (Cramer \& Pschibul, 2017). However, to date, research on the fall break has not been published for a broad audience.

Through a multi-institutional collaboration, our research team aims to address a gap in the literature regarding the impact of a fall break on university students. During the fall of 2015 and around the time of our university's fall reading week (October 12th -15th), we launched the first phase of our project at our university. We collected saliva samples from students immediately before and after their fall break in order to document possible changes in their stress hormones. We also collected saliva samples at the same time points from students attending a university that did not have a fall break. In addition, we collected self-reports of stress through student surveys and focus groups, and we tracked students' time management over the fall break using real-time measures. This study is the first of its kind to attempt to document the broad effects of a fall reading week using mixed methods. Our interdisciplinary team combined qualitative and quantitative approaches to provide the first thorough assessment of the impacts of a fall break on students. This research was approved by the Research Ethics Boards at both universities where data collection occurred, conforming to standards of ethical conduct in research involving human participants.

\section{Research Aims and Research Questions}

Student Experience \& Mental Wellness: How does the fall break affect the workload and stress levels of undergraduate students? How do these students spend their time during the fall break? Are hormonal markers of stress such as cortisol and dehydroepiandrosterone (DHEA) different in undergraduate students who attend a university with 
a fall break than in those at a university without a fall break?

Faculty Experience \& Mental Health: How does the fall break affect the workload and stress levels of instructors?

University Operations: How does the implementation of the fall break affect budgets, staffing, and scheduling within support units?

While we collected data to assess the latter two questions, our focus here will be on the student perspective. We plan to publish the faculty and university operations findings in a forthcoming paper.

\section{Methods}

\section{Data Collection and Analysis}

\section{Student surveys}

During the fall 2015 academic term, all undergraduate students at our university $(N=24$ 940) were invited to participate in a two-part survey focusing on stress. Students completed the survey in October during the week before the fall break $(n=$ 997) and again the week after the fall break $(n=$ 1444). The survey included two validated measures of stress: the Undergraduate Stress Questionnaire (Cohen, Kamarck, \& Mermelstein, 1983), which assesses the number of stressors a student has experienced recently, and the Perceived Stress Scale (Crandall, Preisler, \& Aussprung, 1992), which measures perceived stress in students. We included both measures to explore the sources of stress as well as how students were managing them. We also collected data on a variety of demographic variables. Students were invited to complete a short follow-up survey in January 2016, reflecting on the impact of the fall break on their stress and academic performance over the entire fall semester, including the exam period ( $n=1146)$. All surveys were hosted online; students were emailed with an invitation to participate.

\section{Student time management}

Any students who completed the pre-break survey were able to consent to receive text messages on their phones over the fall break to track how they were spending their time. The immediate reports of activities afforded by this approach are more valid and reliable than retrospective reports (Baddeley, 1979). We had 595 students consent to this part of the study; we sent each of them 12 text messages over the break, asking them to self-report on their current activity. Students received two text messages per day over the break; the messages were delivered at random time points in the day between $8 \mathrm{am}$ and $10 \mathrm{pm}$. To provide clear and reliable categories of behaviour, our text messages asked students to report in which of the following activities they were currently engaged: travelling, schoolwork, work, social time, eating, exercising, leisure, and other.

\section{Hormonal assessment of stress}

In collaboration with a behavioural endocrinology lab at the University of New Orleans, we completed a pilot project that establishes protocols for saliva collection and hormonal analysis around the fall break. We collected saliva samples from first-year male engineering students at our university $(n=11)$ and the comparison university $(n=5)$. In order to control for within-group variance in hormonal output, we included only males in the study and only first-year student engineers from each school due to similar class schedules. The students at the comparison university do not have a fall break but have similar entrance and course requirements to those of our university; they served as a control group.

Participants were given saliva collection microtubes and were asked to identify which day in the week before the fall break that they considered to be most stressful and a day in the week after the break that they considered equally stress-inducing. Similarly, students at the comparison university identified their most stressful day during each of these weeks. Participants were instructed to go to sleep between 10:00-11:30pm on the night before their selected days, to wake at $7 \mathrm{am}$, and to attend all classes on those days. They were asked to collect saliva using 
a modified version of the passive drool technique (Granger et al., 2007): to avoid eating around the time of saliva collection and to drool directly into a microtube at $7 \mathrm{am}, 9 \mathrm{am}, 12 \mathrm{pm}, 3 \mathrm{pm}, 8 \mathrm{pm}$ on each of their two self-identified days. Saliva samples were stored at room temperature before being shipped to the University of New Orleans for hormonal analysis of DHEA and cortisol using standardized enzyme immune assay kits purchased from Salimetrics ${ }^{\circledR}$.

\section{Student focus groups}

Students who completed any of the online surveys in October 2015 and January 2016 were invited to provide their consent to be contacted by email in order to participate in a focus group meant to further explore their experience with the fall break. Of those students who consented to be contacted, 125 students were selected at random and invited to participate in five separate focus groups in late October 2015, and 42 participated. An additional 100 students were invited to participate in a second round of two separate focus groups in early February 2016, and eight students participated. Focus groups were held in a private meeting room on the university campus. Each focus group was facilitated by a member of the research team and lasted approximately 45-60 minutes in length. All focus groups were audio recorded (with participants' permission) to ensure accuracy. The semi-structured focus groups asked participants to discuss such topics as their expectations for how they would spend their time during the fall break, the timing of the fall break itself, and whether the fall break impacted stress levels as well as their ability to be academically successful.

Transcripts were analysed by combining structured as well as open coding processes (Bender \& Ewbank, 1994; Morgan, 1996; Thornberg, 2012). We initiated the coding process with a list of keywords or short phrases that were defined according to our research aims. This was a provisional coding to establish an initial predetermined list of codes prior to a closer analysis of the focus group transcripts. This initial list of keywords was linked to the script used for the focus groups. The phrases used as codes were based on the subject of the research (structural coding).
A member of the research team collaborated with two student partners to produce a first coding of the two focus group transcripts using a provisional list of codes. Each student then began to code all focus group transcripts, and they did so simultaneously. During this process, new codes were added to the initial list as patterns based on repeated ideas, concepts or elements began to emerge, and the student partners compared their codes with one another for inter-rater reliability. These new codes consisted of terms or short phrases to summarize the main theme of a piece of data (descriptive coding). This led to a second list, which consisted of the initial codes plus those arising from the analysis. The student partners used this list to code the remaining transcripts and continued to assess one another's coding process for inter-rater reliability. $\mathrm{NVivo}^{\circledR}$, a qualitative data analysis software program, was used to classify, sort, and arrange information, and to examine relationships in the data.

\section{Results}

\section{Student Surveys}

Due to the small portion of survey respondents that completed both pre-break and post-break surveys $(n$ = 247), all analyses used a between-subjects approach using independent samples t-tests. Scores on the Undergraduate Stress Questionnaire indicated a decrease in the number of stressors over the course of the fall break, $t(2432)=-2.095, p<0.05$. However, scores on the Perceived Stress Scale indicated that perceived stress was higher after the break than before the break, $t(1997)=2.832, p<0.01$.

Frequency analysis of the types of stressors that students most commonly experienced provided some insight into this difference. Namely, the top three stressors before the fall break, summarized by the percentage of respondents reporting each, were: worry about the future $(68.2 \%)$, sitting through a boring class $(68.0 \%)$, and having too little sleep (65.1\%). In contrast, the top three stressors after the break were: having lots of deadlines to meet $(69.4 \%)$, having projects due $(69.0 \%)$, and having a hard 
upcoming week (66.6\%). Thus, post-break stressors were heavily centered around academic responsibilities, while pre-break stressors were more general. The impact of this pattern is discussed below. Analyses of the January survey, which requested students to reflect on the impact of the fall break on their entire fall semester, indicated that the majority of students had a positive perception of the fall break. Of the 1146 respondents, $80 \%$ indicated that the fall break "was a good thing" for them. When asked to evaluate the effect of the fall break on their stress, as compared to Fall 2014 (which did not have a full-week fall break), $69.1 \%$ of respondents indicated that the fall break decreased their stress, $18.2 \%$ reported that it increased their stress, $8.6 \%$ indicated that it did not impact their stress, and 4.1\% were unable to assess the impact on their stress. Although these are retrospective judgments, they demonstrate a consistent perception of a positive impact of the fall break on student stress. Additionally, $53.6 \%$ of respondents judged that the fall break improved their academic performance relative to the previous fall semester, while only $9.8 \%$ judged that it decreased their academic performance. Thus, overall judgments from students reflected a positive perception of the fall break, both in terms of stress and academics. Nonetheless, there is a minority group that reported a negative experience on both measures. We are planning additional analyses to identify the characteristics of this minority, to allow universities to provide appropriate further supports.

When students were asked about the optimal timing of the fall break, responses indicated more variability in satisfaction. Our university's fall break is held relatively early in the term (during the second week of October). While $41.4 \%$ of respondents indicated that the early break was ideal, an overall majority judged that it should be held later in the semester (first week of November: 30.5\%; last week of November: 4.1\%, before December exams: $16.1 \%$ ). Additionally, $7.9 \%$ of respondents judged that it should not be held at all. The remaining respondents $(0.2 \%)$ left this question blank.

\section{Student Time Management}

While we plan to eventually correlate students' behaviour over the fall break with stress scores, at this time, text messages have been analysed only in terms of frequency of behaviour. These frequency analyses provide the total number of text messages received that reported engagement in each type of activity. The most commonly reported activities were schoolwork $(n=674)$ and leisure activities $(n=686)$, while the least commonly reported activities were exercising $(n=70)$ and being at work $(n=170)$.

\section{Hormonal Assessment of Stress}

We analyzed salivary cortisol and DHEA individually using a 2 (university) x 2 (week) mixed-model ANOVA. There was no difference between students at our university (with a fall break) and the comparison university (without a fall break) in their hormonal profiles based on university type or timing around the fall break. However, we also analyzed the ratio of cortisol to DHEA, since some literature suggests that the ratio between these hormones is an informative indicator of mental health (Shirotsuki et al., 2009). Students who experienced a fall break exhibited a lower ratio of cortisol to DHEA after the break, suggesting that students who had a week away from classes seemed to exhibit less stress upon their return to school in comparison to those that did not have a break. It is important to note that the observed post-break difference between the two universities was marginally different $F(1,7)=5.456, p=0.052$. We are currently in the process of collecting further data to support our initial findings.

\section{Student Focus Groups}

Frequency analysis of the focus group transcripts indicated that students were quite mixed in their selfassessment of whether the fall break reduced stress and increased their academic performance. In all five October focus groups, students generally reported a positive personal experience of the fall break. Students 
appreciated the additional time spent at home with family and friends during the Canadian Thanksgiving holiday, the opportunity to rest, engage in leisure activities, catch up on course reading material, and study for midterms scheduled immediately after the break. However, the negative impact of the fall break on course scheduling and the timing of midterms and assignments was also of primary concern among focus group participants. This was reflected in the highest frequency codes, "course scheduling and workload" and "timing of fall break," which are discussed in more detail below.

\section{Course scheduling and workload (frequency: 69)}

Analysis of the five focus groups showed that the highest recurring code related to the scheduling of course assignments, and the shifts in workload that resulted. Upper-year undergraduate students, in particular, reported that in comparison to previous years, the due dates of assignments and the scheduling of midterm examinations were compressed, such that they were scheduled either in the week immediately preceding (October 5-9) or following (October 1923) the fall break. Students reported that this schedule caused a major increase in their perceived stress. As one student stated: "It made me a lot more stressed after [the fall break] because I was trying to do all this stuff during the week before, or during the week off, and then coming back to all these midterms and essays due all at once. It didn't really help me at all. I was just super stressed." Although this perception of the break as a force that compressed assignments and exams in the weeks preceding and following the fall break was common, some students did welcome the break as a "lifesaver" which allowed them an opportunity to rest and prepare for the remainder of the term.

\section{Timing of Fall Break (frequency: 54)}

Another common concern for students related to the timing of the break in the fall term. Although students acknowledged that scheduling the break during the week of the Canadian Thanksgiving holiday (October 12, 2015) limited the number of lost instructional days and gave them additional time with their families, many students considered the timing of the break to be much too early in the term to be effective in meaningfully reducing stress and in supporting academic performance. As one student reported: "I felt last year like the constant stress really helped me be more productive during the days off. Then this year with the break, it really broke my focus to have that much time off instead of focusing on school. I was just getting into the flow of things and right when I got into it, it's like, 'Okay you're off for a week.' It really confused my brain.” Several focus group participants, particularly first-year undergraduate students, expressed concern that the fall break was scheduled too early in the term, and that it significantly disrupted their adjustment to life on a university campus and to the weekly course routine they had begun to establish.

\section{Discussion \& Implications}

Our survey data indicate that compared to stress levels before the break, students experienced higher levels of stress after the fall break, in spite of the fact that they were exposed to a smaller number of stressors. Postbreak stressors were heavily centered around academic responsibilities, while pre-break stressors were more general. Since students' primary responsibility is to academic success, it is possible that the experience of multiple academic-based stressors (e.g., upcoming tests and assignment deadlines) was driving the postbreak increase in perceived stress. Data from the focus groups support this likelihood, with concerns surrounding course scheduling and workload being the most common theme discussed by students.

In spite of the increased stress we observed immediately after the fall break, responses in the follow-up survey in January, in which students reflected on the impact of the fall break on their entire fall semester, indicated that the majority of students had a positive perception of the fall break. Indeed, many respondents felt that it decreased their overall stress levels. One possible explanation for contrasting reports of stress between the October and January 
surveys could stem from the time at which we administered the post-break survey. A substantial portion of respondents indicated that their academic workload immediately before and after the fall break was heavier than usual. It is possible that the break caused a transitory increase in stress, but that this spike was forgotten by the start of the next semester. This pattern could have implications for scheduling decisions made by instructors: being able to avoid an overly dense session of assessments could moderate student stress surrounding the fall break. Several focus group participants suggested that instructors within a department try to coordinate their assessment schedules so as to avoid having several assessments in different courses due in a given week. While the complexities of such a system are considerable, it may be worthwhile to investigate new ways of approaching this issue.

Additionally, our hormonal measures provide some indication that a fall break may, in fact, reduce student stress. Although we consider our preliminary findings promising, we are very cautious in our interpretation, since the data from our pilot study were collected from a subset of the undergraduate population (male, student engineers) and our sample size is limited (16 participants total). We are currently in the process of directing our efforts to recruit more participants in a follow-up study that will assess stress hormones around the timing of the fall break.

Our overall findings suggest that although perceived stress increases immediately after a fall break, students generally feel that the break exerts a positive impact on their stress and academic performance. University administrators may want to consider some factors that could further increase its benefit. For example, a large proportion of students indicated that they would prefer to have the fall break held later in the semester. Many other universities hold the fall break in November, and it would be interesting to assess whether stress levels after a later break correspond to those reported here. Additionally, and as mentioned above, universities may want to introduce a program to prevent excessive evaluation density immediately after the fall break. Note that it is possible that a later fall break could facilitate a less dense evaluation schedule, as instructors would have had more opportunities to evaluate students earlier in the term. It might also be worthwhile for student success and development centres to incorporate stress and time management strategies specific to the fall break within stressreduction programs that are already available to students. At this point, the purpose of the fall break is not clearly delineated by the university. On one hand, the official terminology (fall "break") implies that students should be using the week to take time away from academic responsibilities. On the other hand, the pattern of dense assessment schedules immediately after the break requires that students spend much of their time studying. Our data on time management over the fall break indicate that the most common activities over the fall break are academic and leisure, but it is possible that the university could further support students by providing guidance on effective ways to balance relaxation and academic responsibilities during the week away. Careful consideration of these recommendations could allow universities to ensure that the fall break truly supports students, both academically and in their mental health. Further, we would recommend that any consideration of future changes to programs would make use of the interdisciplinary, mixed methods approaches discussed herein, as they provide a robust evaluation of the complex variation within a university population.

\section{References}

Adlaf, E. M., Gliksman, L., Demers, A., \& NewtonTaylor, B. (2001). The prevalence of elevated psychological distress among Canadian undergraduates: Findings from the 1998 Canadian Campus Survey. Journal of American College Health, 50(2), 67-72. VIEW ITEM

American College Health Association. (2013). American College Health Association National College Health Assessment II: Canadian Reference Group Executive Summary. Hanover, MD: American College Health Association. 
Baddeley, A. (1979). The limitations of human memory: Implications for the design of retrospective surveys. In L. Moss \& $\mathrm{H}$. Goldstein (Eds.), The Recall Method in Social Surveys (pp. 13-27). London, England: University of London Institute of Education.

Bartlett, V. (2014). A roadmap for federal action on student mental health. Ottawa, ON: The Canadian Alliance of Student Associations. VIEW ITEM

Bender, D. E., \& Ewbank, D. (1994). The focus group as a tool for health research: Issues in design and analysis. Health Transition Review, 4(1), 63-80. VIEW ITEM

Booth, R. W., Sharma, D., \& Leader, T. I. (2015). The age of anxiety? It depends where you look: Changes in STAI trait anxiety, 1970-2010. Social Psychiatry and Psychiatric Epidemiology, 51(2), 193-202. VIEW ITEM

Canadian Association of College and University Student Services (2016). Canadian Student Health Data Executive Summary. Toronto, ON: Canadian Association of College and University Student Services.

Chiose, S. (2016, September 8). Reports of mental health issues rising among postsecondary students: Study. The Globe and Mail. VIEW ITEM

Clapham, L., Jahchan, R., Medves, J., Tierney, A., \& Walker, D. (2012). Student mental health and wellness: Framework and recommendations for a comprehensive strategy. (Report of the Principal's Commission on Mental Health). VIEW ITEM

Cohen, S., Kamarck, T., \& Mermelstein, R. (1983). A global measure of perceived stress. Journal of Health and Social Behavior, 24(4), 386-396. VIEW ITEM

Cramer, K., \& Pschibul, R. (2017). Student time usage during fall reading week. Collected Essays on Learning and Teaching, 10, 155-162. VIEW ITEM

Crandall, C. S., Preisler, J. J., \& Aussprung, J. (1992). Measuring life event stress in the lives of college students: The Undergraduate Stress Questionnaire (USQ). Journal of Behavioral Medicine, 15(6), 627-662. VIEW ITEM

Eisenberg, D., Gollust, S. E., Golberstein, E., \& Hefner, J. L. (2007). Prevalence and correlates of depression, anxiety, and suicidality among university students. American Journal of Orthopsychiatry, 77(4), 534-542. VIEW ITEM

Granger, D. A., Kivlighan, K. T., Fortunato, C., Harmon, A. G., Hibel, L. C., Schwartz, E. B., $\&$ Whemboula, G. -L. (2007). Integration of salivary biomarkers into developmental and behaviorally-oriented research: Problems and solutions for collecting specimens. Physiology \& Behavior, 92(4), 583-590. VIEW ITEM

Lunau, K. (2012, September 5). The mental health crisis on campus: Canadian students feel hopeless, depressed, even suicidal. Maclean's. VIEW ITEM

McMaster Daily News (January 15, 2015). McMaster approves full week fall break. VIEW ITEM

Morgan, D. L. (1996). Focus groups. Annual Review of Sociology, 22, 129-152. VIEW ITEM

Pilato, K. (2014). Exploring the impact of a Fall Break on student mental health outcomes (Year 1 Report). St. Catharines, ON: Brock University. VIEW ITEM

Queen's University (n.d.). Identifying and responding to students in distress. VIEW ITEM

Rao, U., Hammen, C., Daley, S. E. (1999). Continuity of depression during the transition to adulthood: A 5-year longitudinal study of young women. Journal of the American 
Academy of Child and Adolescent Psychiatry, 38(7), 908-915. VIEW ITEM

Shirotsuki, K., Izawa, S., Sugaya, N., Yamada, K. C., Ogawa, N., Ouchi, Y., ... Nomura, S. (2009). Salivary cortisol and DHEA reactivity to psychosocial stress in socially anxious males. International Journal of Psychophysiology, 72(2), 198-203. VIEW ITEM

Statistics Canada (2016). Postsecondary enrolments by institution type, registration status, province and sex. VIEW ITEM

Thornberg, R. (2012). Informed grounded theory. Scandinavian Journal of Educational Research, 56(3), 243-259. VIEW ITEM

Universities Canada (n.d). Facts and stats: Overview. VIEW ITEM

\section{Acknowledgements}

This research was supported by grants from the Arts Research Board, McMaster University and the Canadian Association of College and University Student Services. We greatly appreciate the assistance of Bismah Basharat, Arpa Modi, and Joshua Wiener.

\section{Biographies}

Heather Poole is a postdoctoral fellow of the MacPherson Institute for Leadership, Innovation and Excellence in Teaching of McMaster University and currently teaches in the School of Psychology at the University of Ottawa.

Ayesha Khan is an assistant professor in the Department of Psychology, Neuroscience \& Behaviour at McMaster University.

Michael Agnew is a postdoctoral fellow of the MacPherson Institute for Leadership, Innovation and Excellence in Teaching at McMaster University. 


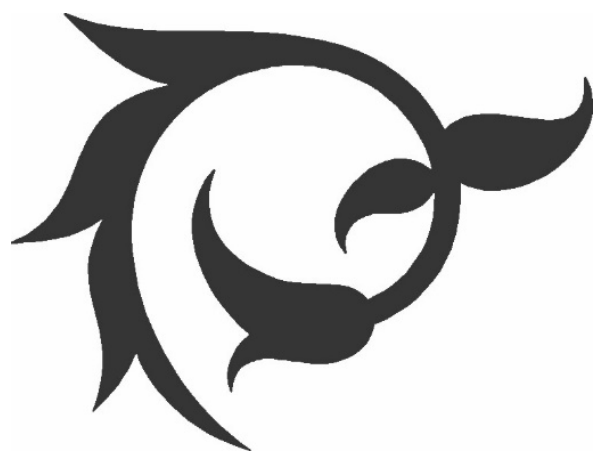

